\title{
A transience condition for a class of one-dimensional symmetric Lévy processes
}

\author{
Nikola Sandrić*
}

\begin{abstract}
In this paper, we give a sufficient condition for the transience for a class of onedimensional symmetric Lévy processes. More precisely, we prove that a one-dimensional symmetric Lévy process with the Lévy measure $\nu(d y)=f(y) d y$ or $\nu(\{n\})=p_{n}$, where the density function $f(y)$ is such that $f(y)>0$ a.e. and the sequence $\left\{p_{n}\right\}_{n \geq 1}$ is such that $p_{n}>0$ for all $n \geq 1$, is transient if

$$
\int_{1}^{\infty} \frac{d y}{y^{3} f(y)}<\infty \text { or } \sum_{n=1}^{\infty} \frac{1}{n^{3} p_{n}}<\infty .
$$

Similarly, we derive an analogous transience condition for one-dimensional symmetric random walks with continuous and discrete jumps.

Keywords: characteristics of a semimartingale; electrical network; Lévy measure; Lévy process; random walk; recurrence; transience.

AMS MSC 2010: 60G17; 60G50; 60G51.

Submitted to ECP on May 15, 2013, final version accepted on August 20, 2013.

Supersedes arXiv: 1308.4626.
\end{abstract}

\section{Introduction}

Let $(\Omega, \mathcal{F}, \mathbb{P})$ be a probability space and let $\left\{L_{t}\right\}_{t>0}$ be a stochastic process on $(\Omega, \mathcal{F}, \mathbb{P})$ taking values in $\mathbb{R}^{d}, d \geq 1$. The process $\left\{L_{t}\right\}_{t>0}$ is called a Lévy process if $L_{0}=0 \mathbb{P}$-a.s., if it has stationary and independent increments and if it has càdlàg paths $\mathbb{P}$-a.s. (that is, if its trajectories are right-continuous with left limits $\mathbb{P}$-a.s.). Having these properties, every Lévy process can be completely and uniquely characterized through the characteristic function of a single random variable $L_{t}, t>0$, that is, by the famous Lévy-Khintchine formula we have

$$
\mathbb{E}\left[\exp \left\{i\left\langle\xi, L_{t}\right\rangle\right\}\right]=\exp \{-t \psi(\xi)\}, \quad t \geq 0,
$$

where

$$
\psi(\xi)=i\langle\xi, b\rangle+\frac{1}{2}\langle\xi, c \xi\rangle+\int_{\mathbb{R}^{d}}\left(1-\exp \{i\langle\xi, y\rangle\}+i\langle\xi, y\rangle 1_{\{|y| \leq 1\}}(y)\right) \nu(d y) .
$$

Here $b$ is a vector in $\mathbb{R}^{d}, c$ is a symmetric nonnegative-definite $d \times d$ matrix and $\nu(d y)$ is a $\sigma$-finite Borel measure on $\mathbb{R}^{d}$ satisfying

$$
\nu(\{0\})=0 \quad \text { and } \quad \int_{\mathbb{R}^{d}} \min \left\{1, y^{2}\right\} \nu(d y)<\infty .
$$

\footnotetext{
${ }^{*}$ Faculty of Civil Engineering, University of Zagreb, Zagreb, Croatia. E-mail: nsandric@grad.hr
} 
The measure $\nu(d y)$, the triplet $(b, c, \nu)$ and the function $\psi(\xi)$ are called the Lévy measure, the Lévy triplet and the characteristic exponent of the Lévy process $\left\{L_{t}\right\}_{t \geq 0}$, respectively. Further, recall that the vector $b$, the matrix $c$ and the Lévy measure $\nu(d y)$ correspond to the deterministic part (shift), the continuous (Brownian) part and the jumping part of the Lévy process $\left\{L_{t}\right\}_{t \geq 0}$, respectively.

In this paper, we consider the transience and recurrence property of Lévy processes. A Lévy process $\left\{L_{t}\right\}_{t \geq 0}$ is said to be transient if

$$
\mathbb{P}\left(\lim _{t \longrightarrow \infty}\left|L_{t}\right|=\infty\right)=1,
$$

and recurrent if

$$
\mathbb{P}\left(\liminf _{t \longrightarrow \infty}\left|L_{t}\right|=0\right)=1 .
$$

It is well known that every Lévy process is either transient or recurrent (see [7, Theorem 35.3]). An equivalent definition (characterization) of the transience and recurrence property of Lévy processes can be given through the sojourn times. A Lévy process $\left\{L_{t}\right\}_{t \geq 0}$ is transient if and only if

$$
\mathbb{E}\left[\int_{0}^{\infty} 1_{\left\{\left|L_{t}\right|<a\right\}}\left(L_{t}\right) d t\right]<\infty \text { for all } a>0 .
$$

Similarly, a Lévy process $\left\{L_{t}\right\}_{t \geq 0}$ is recurrent if and only if

$$
\mathbb{E}\left[\int_{0}^{\infty} 1_{\left\{\left|L_{t}\right|<a\right\}}\left(L_{t}\right) d t\right]=\infty \text { for all } a>0
$$

(see [7, Theorem 35.4]). The above characterizations of the transience and recurrence property are not applicable in most cases. A more operable characterization, by using the nice analytical characterization of Lévy processes through the Lévy-Khintchine formula, has been given by the well-known Chung-Fuchs criterion. A Lévy process $\left\{L_{t}\right\}_{t \geq 0}$ is transient if and only if

$$
\int_{\{|\xi|<a\}} \operatorname{Re}\left(\frac{1}{\psi(\xi)}\right) d \xi<\infty \text { for some } a>0
$$

(see [7, Corollary 37.6 and Remark 37.7]). Again, in many situations this criterion is also not applicable. More precisely, for a given Lévy triplet $(b, c, \nu)$ it is not always easy to compute the integral part of the characteristic exponent as well as the integral appearing in the Chung-Fuchs criterion. According to this, the aim of this paper is to derive a sufficient condition for the transience for Lévy processes in terms of the Lévy triplet. Let us remark that analogous definitions and characterizations of the transience and recurrence property hold also for random walks (see [2, Chapter 4]). Recall that a random walk is a stochastic process $\left\{S_{n}\right\}_{n \geq 0}$ defined on a probability space $(\Omega, \mathcal{F}, \mathbb{P})$ taking values in $\mathbb{R}^{d}, d \geq 1$, defined by $S_{0}:=0$ and $S_{n}:=\sum_{i=1}^{n} J_{i}$, where $\left\{J_{n}\right\}_{n \geq 1}$ is a sequence of i.i.d. random variables called the jumps of $\left\{S_{n}\right\}_{n \geq 0}$.

As already mentioned, in this paper we consider the one-dimensional symmetric case only. Note that, according to [7, Theorem 37.8] and [2, Theorem 4.2.13], the limitation to the one-dimensional case is not too big restriction since it is well known that every $d$-dimensional, $d \geq 3$, Lévy process and random walk are transient. Further, recall that a stochastic process $\left\{X_{t}\right\}_{t \in \mathbb{T}}$ is symmetric if $\left\{X_{t}\right\}_{t \in \mathbb{T}} \stackrel{\mathrm{d}}{=}\left\{-X_{t}\right\}_{t \in \mathbb{T}}$, where $\mathbb{T}=[0, \infty)$ or $\{0,1,2, \ldots\}$ and $\stackrel{\mathrm{d}}{=}$ means that the processes $\left\{X_{t}\right\}_{t \in \mathbb{T}}$ and $\left\{-X_{t}\right\}_{t \in \mathbb{T}}$ have the same finite-dimensional distributions. In the Lévy process case, $\left\{L_{t}\right\}_{t \geq 0}$ is symmetric if and only if $b=0$ and $\nu(d y)$ is a symmetric measure, that is, $\nu(B)=\nu(-B)$ holds for all Borel sets $B \subseteq \mathbb{R}^{d}$ (see [7, Exercise 18.1]), while a random walk is symmetric if and only if its jumps have a symmetric distribution. Now, let us state the main results of this paper. 
Theorem 1.1. Let $\left\{L_{t}\right\}_{t \geq 0}$ be a one-dimensional symmetric Lévy process with the Lévy measure $\nu(d y)=f(y) d y$ or $\nu(\{n\})=p_{n}$, where the density function $f(y)$ is such that $f(y)>0$ a.e. and the sequence $\left\{p_{n}\right\}_{n \geq 1}$ is such that $p_{n}>0$ for all $n \geq 1$. Then, $\left\{L_{t}\right\}_{t \geq 0}$ is transient if

$$
\int_{1}^{\infty} \frac{d y}{y^{3} f(y)}<\infty \text { or } \sum_{n=1}^{\infty} \frac{1}{n^{3} p_{n}}<\infty .
$$

By using [7, Theorem 38.2], the above transience condition can be generalized to the general symmetric case.

Theorem 1.2. Let $\left\{L_{t}^{1}\right\}_{t \geq 0}$ and $\left\{L_{t}^{2}\right\}_{t \geq 0}$ be one-dimensional symmetric Lévy processes with the Lévy measures $\nu_{1}(d y)$ and $\nu_{2}(d y)$. Further, let $\nu_{1}(d y)$ be as in Theorem 1.1 and let it satisfy the condition (1.1). If

$$
\int_{0}^{\infty} y^{2}\left|\nu_{1}-\nu_{2}\right|(d y)<\infty
$$

then the transience property of $\left\{L_{t}^{1}\right\}_{t \geq 0}$ implies the transience property of $\left\{L_{t}^{2}\right\}_{t \geq 0}$. Here $|\cdot|$ denotes the total variation norm on the space of signed measures.

Let us remark that the same transience condition holds also in the case of onedimensional symmetric random walks. More precisely, let $\left\{S_{n}\right\}_{n \geq 0}$ be a one-dimensional symmetric random walk with jumps $\mathbb{P}\left(J_{1} \in d y\right)=f(y) d y$ or $\mathbb{P}\left(J_{1}=n\right)=p_{n}$, where $f(y)>0$ a.e. and $p_{n}>0$ for all $n \geq 1$, then the condition (1.1) implies the transience property of $\left\{S_{n}\right\}_{n \geq 0}$. Also, let us remark that, according to [7, Theorem 38.2] or [8, Lemma 1.2], the assumptions $f(y)>0$ a.e. and $p_{n}>0$ for all $n \geq 1$ can be relaxed. More precisely, it suffices to demand positivity of $f(y)$ and $p_{n}$ on the complement of a compact set.

A simple application of the condition (1.1) is in the class of stable processes. Recall that a one-dimensional symmetric stable Lévy process or a random walk is given by the characteristic exponent $\psi(\xi)=\gamma|\xi|^{\alpha}$ or by the Lévy triplet $(0, c, \nu)$, where $\gamma>0$, $\alpha \in(0,2]$,

$$
c=\left\{\begin{array}{cc}
0, & \alpha<2 \\
2 \gamma, & \alpha=2
\end{array} \quad \text { and } \quad \nu(d y)=\left\{\begin{array}{cc}
\gamma \frac{\alpha 2^{\alpha-1} \Gamma\left(\frac{\alpha+1}{2}\right)}{\pi^{\frac{1}{2}} \Gamma\left(1-\frac{\alpha}{2}\right)}|y|^{-\alpha-1} d y, & \alpha<2 \\
0, & \alpha=2
\end{array}\right.\right.
$$

It is well known, as a consequence of the Chung-Fuchs criterion, that this process is transient if and only if $\alpha<1$. Further, recall that a probability density function of a one-dimensional symmetric stable distribution behaves like $c_{\alpha}|y|^{-\alpha-1}$ when $|y| \longrightarrow \infty$, for $\alpha \in(0,2)$ and

$$
c_{\alpha}=\left\{\begin{array}{cc}
\frac{\gamma}{2}, & \alpha=1 \\
\frac{\gamma}{\pi} \Gamma(\alpha+1) \sin \left(\frac{\pi \alpha}{2}\right), & \alpha \neq 1
\end{array}\right.
$$

(see [7, Remark 14.18]). Now, as a simple consequence of Theorem 1.1, we get a new proof for the transience property of one-dimensional symmetric stable Lévy processes and random walks.

Corollary 1.3. A one-dimensional symmetric stable Lévy process or a random walk is transient if $\alpha<1$.

Note that the above corollary implies that the function $y \longmapsto y^{3}$, appearing in the condition (1.1), is optimal in the class of power functions.

The transience and recurrence property of one-dimensional symmetric Lévy processes in terms of the Lévy triplet has already been studied in the literature. Namely, in 
[7, Theorem 38.3] (see also [8, Theorem 5]) it has been proved that a one-dimensional symmetric Lévy process $\left\{L_{t}\right\}_{t \geq 0}$ with the Lévy measure $\nu(d y)$ is recurrent if

$$
\int_{1}^{\infty}\left(\int_{0}^{y} z \nu(\max \{1, z\}, \infty) d z\right)^{-1} d y=\infty
$$

Intuitively, the condition (1.2) measures the speed of divergence of the second moment of $\nu(d y)$, and, regarding this speed, it concludes the recurrence property. Clearly, if $\nu(d y)$ has finite second moment, then $\left\{L_{t}\right\}_{t \geq 0}$ is recurrent. Thus, if the second moment of $\nu(d y)$ diverges slow enough, then $\left\{L_{t}\right\}_{t \geq 0}$ is recurrent. Similarly, the condition (1.1) measures the speed of divergence of the third moment of $\nu(d y)$. If the third moment of $\nu(d y)$ diverges fast enough, then $\left\{L_{t}\right\}_{t \geq 0}$ is transient.

Recall that a symmetric Borel measure $\rho(d y)$ on $\mathbb{R}$ is unimodal if it is finite outside of any neighborhood of the origin and if $x \longmapsto \rho(x, \infty)$ is a convex function on $(0, \infty)$. Equivalently, a symmetric Borel measure $\rho(d y)$ on $\mathbb{R}$ is unimodal if it is of the form $\rho(d y)=a \delta_{0}(d y)+f(y) d y$, where $0 \leq a \leq \infty$ and the density function $f(y)$ is symmetric, decreasing on $(0, \infty)$ and it satisfies $\int_{|y|>\varepsilon} f(y) d y<\infty$ for all $\varepsilon>0$ (see [7, Chapter 5]). Note that measures with a discrete support are never unimodal. Now, if the Lévy measure $\nu(d y)$ is additionally unimodal, the condition (1.2) is also necessary for the recurrence property (see [7, Theorem 38.3] or [8, Theorem 5]). Also, note that unimodality of the $\nu(d y)$ and finiteness of (1.2) imply that $f(y)>0$ a.e., and the condition (1.2) is stronger than the condition (1.1) (see Section 4 for the proof). Thus, the condition (1.1) is a generalization of the condition (1.2) in the case when the jumping measure is not unimodal. For the necessity of unimodality for the characterization of the transience property by the condition (1.2) see [7, Theorems 38.2, 38.3 and 38.4 and Lemma 38.8] or [8, Theorems 4 and 5] and [9, Theorem 1].

Finally, we give an example where the condition (1.1) is more suitable than the Chung-Fuchs criterion and the condition (1.2). We consider an example of a Lévy process with "multiple indices of stability". Let $\left\{L_{t}\right\}_{t \geq 0}$ be a one-dimensional symmetric Lévy process with the Lévy measure $\nu(\{n\})=p_{n}$, where $p_{2 n}=(2 n)^{-\alpha-1}$ and $p_{2 n-1}=(2 n-1)^{-\beta-1}$ for $n \geq 1$ and $\alpha, \beta \in(0, \infty)$. For a continuous version of such process it suffices to interpolate the points $\left\{\left(i, p_{i}\right): i \in \mathbb{Z}\right\}$. Now, clearly, if $\alpha<1$ and $\beta<1$, then the condition (1.1) implies the transience of $\left\{L_{t}\right\}_{t \geq 0}$. On the other hand, since $\nu(d y)$ is not unimodal, the condition (1.2) is not applicable, and the application of the Chung-Fuchs criterion leads to a non-trivial computation. Also, let us remark that the same example shows that the condition (1.1) is only sufficient for the transience. Indeed, assume that $\alpha<1$ and $\beta \geq 1$. Then, since $\beta \geq 1$, the condition (1.1) fails to hold. On the other hand, since $\alpha<1,\left\{L_{t}\right\}_{t \geq 0}$ is transient (see Section 4 for the proof).

Now, we explain our strategy of proving Theorem 1.1. The proof is divided in three steps. In the first step, by using electrical networks techniques, we prove Theorem 1.1 in the case of a random walk with discrete jumps. In the second step, we prove Theorem 1.1 in the case of a random walk $\left\{S_{n}\right\}_{n \geq 0}$ with continuous jumps $\mathbb{P}\left(J_{1} \in d y\right)=f(y) d y$. More precisely, for $\delta>0$ we define a discretization of $\left\{S_{n}\right\}_{n \geq 0}$ as a random walk $\left\{S_{n}^{\delta}\right\}_{n \geq 0}$ on $\delta \mathbb{Z}$ with the jump distribution $\mathbb{P}\left(J_{1}^{\delta}=\delta n\right):=\int_{\delta n-\frac{\delta}{2}}^{\delta n+\frac{\delta}{2}} f(y) d y, n \in \mathbb{Z}$. Next, by an "approximation approach" we prove that all the random walks $\left\{S_{n}^{\delta}\right\}_{n \geq 0}, \delta>0$, are either transient or recurrent at the same time and their transience and recurrence property is equivalent with the transience and recurrence property of $\left\{S_{n}\right\}_{n \geq 0}$. Finally, by using the first step, we prove that the condition (1.1) for $\left\{S_{n}\right\}_{n \geq 0}$ implies the transience property of $\left\{S_{n}^{1}\right\}_{n \geq 0}$. And this accomplishes the proof of the second step. At the end, in the last step, we consider the case of Lévy processes. By using [7, Theorem 38.2], it suffices to consider the situation of a compound Poisson process. Now, the proof follows from the first and second step. This accomplishes the proof of Theorem 1.1. 
The paper is organized as follows. In Section 2, we give a proof of Theorem 1.1 for the case of discrete jumps. In Section 3, by using the results from Section 2, we proof Theorem 1.1 for the case of continuous jumps. Finally, in Section 4, we discuss some properties of the condition (1.1).

\section{Discrete case}

In this section, we prove the main step of the proof of Theorem 1.1. More precisely, we derive a sufficient condition for the transience for one-dimensional symmetric random walks on $\mathbb{Z}$.

Theorem 2.1. Let $\left\{S_{n}\right\}_{n \geq 0}$ be a one-dimensional symmetric random walk on $\mathbb{Z}$ with jumps $\mathbb{P}\left(J_{1}=n\right)=p_{n}$, where the sequence $\left\{p_{n}\right\}_{n \geq 1}$ is such that $p_{n}>0$ for all $n \geq 1$. Then the random walk $\left\{S_{n}\right\}_{n \geq 0}$ is transient if

$$
\sum_{n \geq 1} \frac{1}{n^{3} p_{n}}<\infty
$$

Note that the same transience condition also holds in the case of a one-dimensional symmetric Lévy process $\left\{L_{t}\right\}_{t \geq 0}$ with a discrete supported Lévy measure $\nu(\{n\})=p_{n}$, where $p_{n}>0$ for all $n \geq 1$. Indeed, first note that

$$
\left\{L_{t}\right\}_{t \geq 0} \stackrel{\mathrm{d}}{=}\left\{S_{P_{t}}\right\}_{t \geq 0},
$$

where $\left\{S_{n}\right\}_{n \geq 0}$ is a random walk with jumps $\mathbb{P}\left(J_{1}=n\right):=\frac{1}{\nu(\mathbb{Z})} p_{n}$ and $\left\{P_{t}\right\}_{t \geq 0}$ is the Poisson process with parameter $\nu(\mathbb{Z})$ independent of $\left\{S_{n}\right\}_{n \geq 0}$. Now, the desired result follows from the definition of the transience in terms of sojourn times.

The proof of Theorem 2.1 is based on techniques and results from electrical networks. Let us introduce some notation we need. A graph is a pair $G=(V(G), E(G))$ where $V(G)$ is a set of vertices and $E(G)$ is a symmetric subset of $V(G) \times V(G)$, called the edge set. By symmetry we mean that $(u, v) \in E(G)$ if and only if $(v, u) \in E(G)$. For two vertices $u, v \in V(G)$ such that $(u, v) \in E(G)$, we say that $u$ and $v$ are adjacent and write $u \sim v$ and by $e_{u v}$ we denote the edge which connects them. A path in a graph is a sequence of vertices where each successive pair of vertices is an edge in the graph. A graph is connected if there is a path from any of its vertices to any other. A network is a pair $N=(G, c)$, where $G$ is a connected graph and $c$ is a function $c: E(G) \longrightarrow[0, \infty)$ called conductance. In the sequel we assume that a network $N$ satisfies

$$
c(u):=\sum_{v \sim u} c\left(e_{u v}\right)<\infty
$$

for all $u \in V(G)$. A random walk on a network $N$ is a time-homogeneous Markov chain $\left\{X_{n}\right\}_{n \geq 0}$ with the state space $V(G)$ and transition kernel

$$
q_{u v}:=\mathbb{P}\left(X_{1}=v \mid X_{0}=u\right)=\left\{\begin{array}{cc}
\frac{c\left(e_{u v}\right)}{c(u)}, & u \sim v \\
0, & \text { otherwise }
\end{array}\right.
$$

Note that the Markov chain $\left\{X_{n}\right\}_{n \geq 0}$ is irreducible (that is, $\sum_{n=1}^{\infty} \mathbb{P}\left(X_{n}=v \mid X_{0}=u\right)>0$ for all $u, v \in V(G)$ ) and it is reversible (that is, there exists a nontrivial measure $\pi(d y)$ on $V(G)$, such that $\pi(u) q_{u v}=\pi(v) q_{v u}$ for all $u, v \in V(G)$ ). Indeed, irreducibility easily follows from connectedness of the graph $G$ and for the reversibility measure we can take $\pi:=c$. Also, let us remark that to every irreducible and reversible time-homogeneous Markov chain on a discrete state space $S$ given by the transition kernel $q_{u v}, u, v \in S$, and a reversibility measure $\pi(d y)$ we can join a network $N=(G, c)$. Indeed, put $V(G)=S$, 
the vertices $u$ and $v$ are adjacent if $q_{u v}>0$, the graph $G$ is connected because of irreducibility of the corresponding Markov chain and the conductance is defined by $c\left(e_{u v}\right)=\pi(u) q_{u v}$.

Further, let $u_{0} \in V(G)$ be an arbitrary vertex of the network $N$. A flow from $u_{0}$ to $\infty$ is a function $\theta: V(G) \times V(G) \longrightarrow \mathbb{R}$ such that $\theta(u, v)=0$ unless $u \sim v, \theta(u, v)=-\theta(v, u)$ for all $u, v \in V(G)$ and $\sum_{v \in V(G)} \theta(u, v)=0$ if $u \neq u_{0}$. We call the flow a unit flow if $\sum_{u \in V(G)} \theta\left(u_{0}, u\right)=1$. The energy of the flow is defined by

$$
\mathcal{E}(\theta)=\frac{1}{2} \sum_{u \sim v} \frac{\theta^{2}(u, v)}{c\left(e_{u v}\right)}
$$

Next, recall that a state $u$ of a time-homogeneous Markov chain $\left\{X_{n}\right\}_{n \geq 0}$ on a discrete state space $S$ is called transient if $\sum_{n=1}^{\infty} \mathbb{P}\left(X_{n}=u \mid X_{0}=u\right)<\infty$ and it is called recurrent if $\sum_{n=1}^{\infty} \mathbb{P}\left(X_{n}=u \mid X_{0}=u\right)=\infty$. If every state is transient (resp. recurrent) the chain itself is called transient (resp. recurrent). It is well known that every irreducible Markov chain is either transient or recurrent (see [6, Theorem 8.1.2]). Finally, the main tool for proving Theorem 2.1 is given in the following theorem.

Theorem 2.2. [5, Theorem 1] Random walk on a network $N$ is transient if and only if there is a unit flow on $N$ of finite energy from some vertex to $\infty$.

Proof of Theorem 2.1. First, note that, according to [8, Lemma 1.2], without loss of generality we can assume that $p_{0}>0$. Thus, $\left\{S_{n}\right\}_{n \geq 0}$ is a random walk on the network $N=(G, c)$, where $G=(V(G), E(G))=(\mathbb{Z}, \mathbb{Z} \times \mathbb{Z})$ and $c\left(e_{u v}\right)=p_{|v-u|}$. Now, following the ideas from [3, Theorem 1], we construct a unit flow from 0 to $\infty$ for the random walk $\left\{S_{n}\right\}_{n \geq 0}$ such that the corresponding energy is bounded from above by (2.1). Then the desired result follows from Theorem 2.2. First, let us partition the set of vertices $V(G)=\mathbb{Z}$ on the sets $B_{0}=\{0\}, B_{i}=\left\{2^{i-1}, 2^{i-1}+1, \ldots, 2^{i}-1\right\}$ and $B_{-i}=\left\{-2^{i}+\right.$ $\left.1, \ldots,-2^{i-1}-1,-2^{i-1}\right\}, i \geq 1$, and let us define a unit flow $\theta: V(G) \times V(G) \longrightarrow \mathbb{R}$ from 0 to $\infty$ in the following way. For $u \in B_{i}$ and $v \in B_{j}$ define

$$
\theta(u, v):=\left\{\begin{array}{cc}
\frac{1}{2}, & i=0 \text { and } j=-1 \text { or } j=1 \\
0, & i=j \text { or }|i-j| \geq 2 \\
2^{-2|i|}, & 0<i<j=i+1 \text { or } j<j+1=i<0 .
\end{array}\right.
$$

Recall that flow has to be antisymmetric, hence we define $\theta(v, u):=-\theta(u, v)$. Next, note that

$$
\sum_{v \in \mathbb{Z}} \theta(1, v)=\theta(1,0)+\theta(1,2)+\theta(1,3)=-\frac{1}{2}+\frac{1}{4}+\frac{1}{4}=0
$$

and analogously $\sum_{v \in \mathbb{Z}} \theta(-1, v)=0$. Further, for $u \in B_{i}, i \geq 2$, we have

$$
\sum_{v \in \mathbb{Z}} \theta(u, v)=\sum_{v \in B_{i-1}} \theta(u, v)+\sum_{v \in B_{i+1}} \theta(u, v)=-2^{i-2} 2^{-2(i-1)}+2^{i} 2^{-2 i}=0
$$

and analogously for $u \in B_{i}, i \leq-2$, we have

$$
\sum_{v \in \mathbb{Z}} \theta(u, v)=0
$$

According to this, $\theta$ is a flow from 0 to $\infty$. Finally, since $\sum_{v \in \mathbb{Z}} \theta(0, v)=\theta(0,1)+\theta(0,-1)=1$, $\theta$ is a unit flow from 0 to $\infty$. Now, let us prove that the energy of the flow $\theta$ is bounded 
A transience condition for a class of one-dimensional symmetric Lévy processes

from above by (2.1). We have

$$
\begin{aligned}
\mathcal{E}(\theta)= & \frac{1}{2} \sum_{u \sim v} \frac{\theta^{2}(u, v)}{c\left(e_{u v}\right)}=\frac{1}{2} \sum_{(u, v) \in E(G)} \frac{\theta^{2}(u, v)}{p_{|v-u|}} \\
= & \frac{1}{2} \sum_{u \geq 0, v \geq 0, u \neq v} \frac{\theta^{2}(u, v)}{p_{|v-u|}}+\frac{1}{2} \sum_{u \geq 0, v<0} \frac{\theta^{2}(u, v)}{p_{|v-u|}} \\
& +\frac{1}{2} \sum_{u<0, v \geq 0} \frac{\theta^{2}(u, v)}{p_{|v-u|}}+\frac{1}{2} \sum_{u<0, v<0, u \neq v} \frac{\theta^{2}(u, v)}{p_{|v-u|}} .
\end{aligned}
$$

Note that, from the symmetry of the distribution $\left\{p_{n}\right\}_{n \in \mathbb{Z}}$ and the definition of the flow $\theta$, the second and the third therm equal $\frac{1}{8 p_{1}}$. Next, again from the symmetry of the distribution $\left\{p_{n}\right\}_{n \in \mathbb{Z}}$ and the symmetry of the function $\theta^{2}$, we have

$$
\begin{aligned}
\mathcal{E}(\theta) & \leq \frac{1}{4 p_{1}}+\sum_{u \geq 0, v \geq 0} \frac{\theta^{2}(u, v)}{p_{|v-u|}} \\
& =\frac{1}{4 p_{1}}+\sum_{u \geq 0, w \geq 0} \frac{\theta^{2}(u, u+w)}{p_{w}}+\sum_{u \leq 0, w \leq 0} \frac{\theta^{2}(u, u+w)}{p_{|w|}} \\
& =\frac{1}{4 p_{1}}+2 \sum_{u \geq 0, w \geq 0} \frac{\theta^{2}(u, u+w)}{p_{w}} .
\end{aligned}
$$

Note that $\theta(u, u+w)=0$ when $u+w \geq 4 u$, except for $u=0$ and $w=1$. Thus

$$
\begin{aligned}
\mathcal{E}(\theta) & \leq \frac{3}{4 p_{1}}+2 \sum_{w \geq 2, u \geq\left\lceil\frac{w}{3}\right\rceil} \frac{\theta^{2}(u, u+w)}{p_{w}} \\
& =\frac{3}{4 p_{1}}+\frac{1}{8 p_{2}}+2 \sum_{w \geq 2, u \geq\left\lceil\frac{w}{3}\right\rceil, u \neq 1} \frac{\theta^{2}(u, u+w)}{p_{w}},
\end{aligned}
$$

where $\lceil x\rceil$ denotes the smallest integer not less than $x$. Now, since for $u \in B_{i}, i \geq 2$, (that is, for $u \geq 2$ ), we have $\theta^{2}(u, v) \leq\left(2^{-2(i-1)}\right)^{2}=16\left(2^{i}\right)^{-4} \leq 16 u^{-4}$, then

$$
\sum_{u \geq\left\lceil\frac{w}{3}\right\rceil, u \neq 1}^{\infty} \theta^{2}(u, u+w) \leq\left\{\begin{array}{cc}
\int_{\left\lceil\frac{w}{3}\right\rceil-1}^{\infty} \frac{16}{x^{4}} d x=\frac{16}{3\left(\left\lceil\frac{w}{3}\right\rceil-1\right)^{3}} \leq \frac{144}{(w-3)^{3}}, & w \geq 4 \\
\int_{1}^{\infty} \frac{16}{x^{4}} d x=\frac{16}{3}, & w=2,3 .
\end{array}\right.
$$

This yields

$$
\begin{aligned}
\mathcal{E}(\theta) & \leq \frac{3}{4 p_{1}}+\frac{1}{8 p_{2}}+2 \sum_{w \geq 2} \frac{1}{p_{w}} \sum_{u \geq\left\lceil\frac{w}{3}\right\rceil, u \neq 1} \theta^{2}(u, u+w) \\
& \leq \frac{3}{4 p_{1}}+\frac{1}{8 p_{2}}+\frac{32}{3 p_{2}}+\frac{32}{3 p_{3}}+288 \sum_{w \geq 4} \frac{1}{(w-3)^{3} p_{w}} .
\end{aligned}
$$

This accomplishes the proof of Theorem 2.1.

\section{Continuous case}

In this section, we prove Theorem 1.1 in the case of continuous jumps. As in the case of discrete jumps, the main step is to consider the random walk case. 
A transience condition for a class of one-dimensional symmetric Lévy processes

Theorem 3.1. Let $\left\{S_{n}\right\}_{n \geq 0}$ be one-dimensional symmetric random walk with jumps $\mathbb{P}\left(J_{1} \in d y\right)=f(y) d y$, where the probability density function $f(y)$ is such that $f(y)>0$ a.e. Then the random walk $\left\{S_{n}\right\}_{n \geq 0}$ is transient if

$$
\int_{1}^{\infty} \frac{d y}{y^{3} f(y)}<\infty .
$$

Again, similarly as in the case of discrete jumps, the transience condition for the Lévy process case can be easily derived from the random walk case. Indeed, let $\left\{L_{t}\right\}_{t \geq 0}$ be a one-dimensional symmetric Lévy process with the Lévy measure $\nu(d y)=f(y) d y$, where the density $f(y)$ is such that $f(y)>0$ a.e. Then, first note that, according to [7, Theorem 38.2], without loss of generality we can assume that $\nu(\mathbb{R})<\infty$. Thus,

$$
\left\{L_{t}\right\}_{t \geq 0} \stackrel{\mathrm{d}}{=}\left\{S_{P_{t}}\right\}_{t \geq 0}
$$

where $\left\{S_{n}\right\}_{n \geq 0}$ is a random walk with continuous jumps $\mathbb{P}\left(J_{1} \in d y\right):=\frac{1}{\nu(\mathbb{R})} f(y) d y$ and $\left\{P_{t}\right\}_{t \geq 0}$ is the Poisson process with parameter $\nu(\mathbb{R})$ independent of $\left\{S_{n}\right\}_{n \geq 0}$. Now, the desired result follows from the definition of the transience in terms of sojourn times.

Before the proof of Theorem 3.1, we need some auxiliary results. Let $B \subseteq \mathbb{R}^{d}$ be an arbitrary Borel set and let us denote by $\mathbb{D}\left(\mathbb{R}^{d}\right)$ the space of $\mathbb{R}^{d}$-valued càdlàg functions equipped with the Skorohod topology. Define the set of recurrent paths by

$$
R(B):=\left\{\omega \in \mathbb{D}\left(\mathbb{R}^{d}\right): \forall n \in \mathbb{N}, \exists t \geq n \text { such that } \omega(t) \in B\right\},
$$

and the set of transient paths by

$$
T(B):=\left\{\omega \in \mathbb{D}\left(\mathbb{R}^{d}\right): \exists s \geq 0 \text { such that } \omega(t) \notin B, \forall t \geq s\right\} .
$$

In the following proposition, we characterize the transience and recurrence property of Lévy process in terms of càdlàg paths.

Proposition 3.2. Let $\mathbf{L}=\left\{L_{t}\right\}_{t \geq 0}$ be an $\mathbb{R}^{d}$-valued Lévy process. Then, $\boldsymbol{L}$ is transient if and only if $\mathbb{P}_{\boldsymbol{L}}\left(T\left(B_{a}\right)\right)=1$ for all $a>0$, and it is recurrent if and only if $\mathbb{P}_{\boldsymbol{L}}\left(R\left(B_{a}\right)\right)=1$ for all $a>0$, where $B_{a}$ denotes the open ball of radius a around the origin.

Proof. The proof follows directly from the definition of the transience and recurrence properties.

Now, let us recall the notion of characteristics of a semimartingale (see [4]). Let $\left(\Omega, \mathcal{F},\left\{\mathcal{F}_{t}\right\}_{t \geq 0}, \mathbb{P},\left\{S_{t}\right\}_{t \geq 0}\right),\left\{S_{t}\right\}_{t \geq 0}$ in the sequel, be a one-dimensional semimartingale and let $h: \mathbb{R} \longrightarrow \mathbb{R}$ be a truncation function (that is, a continuous bounded function such that $h(x)=x$ in a neighborhood of the origin). We define two processes

$$
\check{S}(h)_{t}:=\sum_{s \leq t}\left(\Delta S_{s}-h\left(\Delta S_{s}\right)\right) \quad \text { and } \quad S(h)_{t}:=S_{t}-\check{S}(h)_{t},
$$

where the process $\left\{\Delta S_{t}\right\}_{t \geq 0}$ is defined by $\Delta S_{t}:=S_{t}-S_{t-}$ and $\Delta S_{0}:=S_{0}$. The process $\left\{S(h)_{t}\right\}_{t \geq 0}$ is a special semimartingale. Hence, it admits the unique decomposition

$$
S(h)_{t}=S_{0}+M(h)_{t}+B(h)_{t}
$$

where $\left\{S(h)_{t}\right\}_{t \geq 0}$ is a local martingale and $\left\{S(h)_{t}\right\}_{t \geq 0}$ is a predictable process of bounded variation. 
A transience condition for a class of one-dimensional symmetric Lévy processes

Definition 3.3. Let $\left\{S_{t}\right\}_{t>0}$ be a semimartingale and let $h: \mathbb{R} \longrightarrow \mathbb{R}$ be the truncation function. Furthermore, let $\left\{B(h)_{t}\right\}_{t \geq 0}$ be the predictable process of bounded variation appearing in (3.2), let $N(\omega, d s, d y)$ be the compensator of the jump measure

$$
\mu(\omega, d s, d y)=\sum_{s: \Delta S_{s}(\omega) \neq 0} \delta_{\left(s, \Delta S_{s}(\omega)\right)}(d s, d y)
$$

of the process $\left\{S_{t}\right\}_{t \geq 0}$ and let $\left\{C_{t}\right\}_{t \geq 0}$ be the quadratic co-variation process for $\left\{S_{t}^{c}\right\}_{t \geq 0}$ (continuous martingale part of $\left\{S_{t}\right\}_{t \geq 0}$ ), that is,

$$
C_{t}=\left\langle S_{t}^{c}, S_{t}^{c}\right\rangle
$$

Then $(B, C, N)$ is called the characteristics of the semimartingale $\left\{S_{t}\right\}_{t \geq 0}$ (relative to $h(x))$. If we put $\tilde{C}(h)_{t}:=\left\langle M(h)_{t}, M(h)_{t}\right\rangle$, where $\left\{M(h)_{t}\right\}_{t \geq 0}$ is the local martingale appearing in (3.2), then $(B, \tilde{C}, N)$ is called the modified characteristics of the semimartingale $\left\{S_{t}\right\}_{t \geq 0}$ (relative to $h(x)$ ).

Proposition 3.4. Let $\boldsymbol{S}=\left\{S_{n}\right\}_{n \geq 0}$ be a one-dimensional random walk with continuous jumps $\mathbb{P}\left(J_{1} \in d y\right)=f(y) d y$. For $\delta>0$, let $\boldsymbol{S}^{\delta}=\left\{S_{n}^{\delta}\right\}_{n \geq 0}$ be a random walk on $\delta \mathbb{Z}$ with discrete jumps

$$
\mathbb{P}\left(J_{1}^{\delta}=\delta n\right)=\int_{\delta n-\frac{\delta}{2}}^{\delta n+\frac{\delta}{2}} f(y) d y, \quad n \in \mathbb{Z} .
$$

Further, let $\left\{P_{t}\right\}_{t \geq 0}$ be the Poisson process with parameter 1 independent of $\boldsymbol{S}$ and $\boldsymbol{S}^{\delta}$, $\delta>0$, and let $\overline{\boldsymbol{S}}:=\left\{S_{P_{t}}\right\}_{t \geq 0}$ and $\overline{\boldsymbol{S}}^{\delta}:=\left\{S_{P_{t}}^{\delta}\right\}_{t \geq 0}$. Then $\overline{\boldsymbol{S}}^{\delta} \stackrel{d}{\longrightarrow} \overline{\boldsymbol{S}}$ when $\delta \longrightarrow 0$, where $\stackrel{d}{\longrightarrow}$ denotes the convergence in $\mathbb{D}\left(\mathbb{R}^{d}\right)$ with respect to the Skorohod topology, and all the random walks $\boldsymbol{S}^{\delta}, \delta>0$, are either transient or recurrent at the same time and this transience and recurrence dichotomy is equivalent with the transience and recurrence dichotomy of the random walk $\boldsymbol{S}$.

Proof. Clearly, $\overline{\mathbf{S}}$ and $\overline{\mathbf{S}}^{\delta}, \delta>0$, are processes of bounded variation. Thus, they are semimartingales. Further, let $h(x)$ be the truncation function and let $(B, \tilde{C}, N)$ and $\left(B^{\delta}, \tilde{C}^{\delta}, N^{\delta}\right), \delta>0$, be the modified characteristics of $\overline{\mathbf{S}}$ and $\overline{\mathbf{S}}^{\delta}, \delta>0$, respectively. Now, since $\overline{\mathbf{S}}$ and $\overline{\mathbf{S}}^{\delta}$ are Lévy processes, by [4, Proposition 2.17 and Corollary II.4.19], their (modified) characteristics are exactly the corresponding Lévy triplets, that is,

$$
B_{t}=t \mathbb{E}\left[h\left(J_{1}\right)\right], \quad N(d s, d y)=d s \mathbb{P}\left(J_{1} \in d y\right), \quad \tilde{C}_{t}=t \mathbb{E}\left[h^{2}\left(J_{1}\right)\right]
$$

and

$$
B_{t}^{\delta}=t \mathbb{E}\left[h\left(J_{1}^{\delta}\right)\right], \quad N^{\delta}(d s, d y)=d s \mathbb{P}\left(J_{1}^{\delta} \in d y\right), \quad \tilde{C}_{t}^{\delta}=t \mathbb{E}\left[h^{2}\left(J_{1}^{\delta}\right)\right] .
$$

According to this, in order to prove the desired convergence, by [4, Theorem VIII.2.17], it suffices to show that

$$
\sup _{s \leq t}\left|B_{s}^{\delta}-B_{s}\right| \longrightarrow 0, \quad \tilde{C}_{t}^{\delta} \longrightarrow \tilde{C}_{t} \quad \text { and } \quad \int_{[0, t] \times \mathbb{R}} g(y) N^{\delta}(d s, d y) \longrightarrow \int_{[0, t] \times \mathbb{R}} g(y) N(d s, d y)
$$

when $\delta \longrightarrow 0$ for all $t \geq 0$ and for every bounded and continuous function $g(x)$ vanishing in a neighborhood of the origin. Clearly, in order to prove the above convergences, it suffices to show that

$$
\mathbb{E}\left[g\left(J_{1}^{\delta}\right)\right] \longrightarrow \mathbb{E}\left[g\left(J_{1}\right)\right]
$$

when $\delta \longrightarrow 0$ for every bounded and continuous function $g(x)$. But this fact easily follows from [1, Theorem 2.1], definition of the jumps $\left\{J_{n}^{\delta}\right\}_{n \geq 0}, \delta>0$, and continuity of the jumps $\left\{J_{n}\right\}_{n \geq 0}$. 
A transience condition for a class of one-dimensional symmetric Lévy processes

Now, we prove the second part of the proposition. Let $\delta_{0}>0$ be arbitrary. By completely the same arguments as above, we have $\overline{\mathbf{S}}^{\delta} \stackrel{\mathrm{d}}{\longrightarrow} \overline{\mathbf{S}}^{\delta_{0}}$ when $\delta \longrightarrow \delta_{0}$. Next, let $a>0$ be arbitrary, then $T\left(B_{a}\right)=R\left(B_{a}\right)^{c}, R\left(B_{a}\right)$ is open in $\mathrm{D}(\mathbb{R})$ and $\partial R\left(B_{a}\right) \subseteq$ $R\left(B_{a+\varepsilon}\right) \backslash R\left(B_{a-\varepsilon}\right)$, where $\partial R\left(B_{a}\right)$ denotes the boundary of the set $R\left(B_{a}\right)$ and $0<\varepsilon<a$. Thus, by Proposition 3.2, we have

$$
\mathbb{P}_{\overline{\mathbf{s}}^{\delta_{0}}}\left(\partial R\left(B_{a}\right)\right) \leq \mathbb{P}_{\overline{\mathbf{s}}^{\delta_{0}}}\left(R\left(B_{a+\varepsilon}\right)\right)-\mathbb{P}_{\overline{\mathbf{s}}^{\delta_{0}}}\left(R\left(B_{a-\varepsilon}\right)\right)=0
$$

for all $a>0$. Hence, the sets $T\left(B_{a}\right)$ and $R\left(B_{a}\right), a>0$, are continuity sets for $\mathbb{P}_{\overline{\mathbf{s}}^{\delta_{0}}}$. Now, by [1, Theorem 2.1], this yields

$$
\lim _{\delta \longrightarrow \delta_{0}} \mathbb{P}_{\overline{\mathbf{s}}^{\delta}}\left(T\left(B_{a}\right)\right)=\mathbb{P}_{\overline{\mathbf{s}}^{\delta_{0}}}\left(T\left(B_{a}\right)\right)
$$

for all $a>0$. Hence, for all $a>0$, the function

$$
\delta \longmapsto \mathbb{P}_{\overline{\mathbf{s}}^{\delta}}\left(T\left(B_{a}\right)\right)
$$

is continuous on $(0, \infty)$. According to this, by Proposition 3.2, $\mathbb{P}_{\overline{\mathbf{s}}^{\delta}}\left(T\left(B_{a}\right)\right)=1$ for all $\delta>0$ and all $a>0$, or $\mathbb{P}_{\overline{\mathbf{s}}^{\delta}}\left(T\left(B_{a}\right)\right)=0$ for all $\delta>0$ and all $a>0$. This means, again by Proposition 3.2, that all the random walks $\mathbf{S}^{\delta}, \delta>0$, are either transient or recurrent at the same time.

Finally, by completely the same arguments as above, $\mathbb{P}_{\overline{\mathbf{s}}}\left(\partial T\left(B_{a}\right)\right)=0$ for all $a>0$. Then, again by [1, Theorem 2.1], we have

$$
\lim _{\delta \longrightarrow 0} \mathbb{P}_{\overline{\mathbf{s}}^{\delta}}\left(T\left(B_{a}\right)\right)=\mathbb{P}_{\overline{\mathbf{s}}}\left(T\left(B_{a}\right)\right)
$$

for all $a>0$. Thus, by Proposition 3.2, the transience and recurrence property of the random walks $\mathbf{S}^{\delta}, \delta>0$, is equivalent with the transience and recurrence property of the random walk $\mathbf{S}$.

At the end, we prove Theorem 3.1.

Proof of Theorem 3.1. Let $\left\{S_{n}^{1}\right\}_{n \geq 0}$ be a random walk on $\mathbb{Z}$ with discrete jumps

$$
\mathbb{P}\left(J_{1}^{1}=n\right):=\int_{n-\frac{1}{2}}^{n+\frac{1}{2}} f(y) d y, \quad n \in \mathbb{Z}
$$

Next, by the Jensen's inequality, we have

$$
\int_{\frac{1}{2}}^{\infty} \frac{d y}{y^{3} f(y)}=\sum_{n=1}^{\infty} \int_{n-\frac{1}{2}}^{n+\frac{1}{2}} \frac{d y}{y^{3} f(y)} \geq \sum_{n=1}^{\infty} \frac{1}{\left(n+\frac{1}{2}\right)^{3} \int_{n-\frac{1}{2}}^{n+\frac{1}{2}} f(y) d y}=\sum_{n=1}^{\infty} \frac{1}{\left(n+\frac{1}{2}\right)^{3} \mathbb{P}\left(J_{1}^{1}=n\right)}
$$

Thus, by Theorem 2.1, the random walk $\left\{S_{n}^{1}\right\}_{n \geq 0}$ is transient. Now, the desired result follows from Proposition 3.4.

\section{Some remarks on the main results}

In this section, we discus some properties of the condition (1.1) we mentioned in Section 1. First, we prove that, under the assumption of unimodality, the condition (1.2) is stronger than the condition (1.1). Recall that a one-dimensional symmetric Lévy measure $\nu(d y)$ is unimodal if it is of the form $\nu(d y)=f(y) d y$, where the density function 
$f(y)$ is symmetric, decreasing on $(0, \infty)$ and it satisfies $\int_{|y|>\varepsilon} f(y) d y<\infty$ for all $\varepsilon>0$. Let us fix $y_{0}>1$, then, by the Fubini's theorem, for all $y \geq y_{0}$ we have

$$
\begin{aligned}
\int_{0}^{y} z \nu(\max \{1, z\}, \infty) d z & =\int_{0}^{y} z \int_{\max \{1, z\}}^{\infty} f(u) d u d z \\
& =\int_{0}^{1} z \int_{1}^{\infty} f(u) d u d z+\int_{1}^{y} z \int_{z}^{\infty} f(u) d u d z \\
& =\frac{1}{2} \int_{1}^{\infty} f(u) d u+\int_{1}^{y} z \int_{z}^{\infty} f(u) d u d z \\
& =\frac{1}{2} \int_{1}^{y} u^{2} f(u) d u+\frac{y^{2}}{2} \int_{y}^{\infty} f(u) d u \\
& \geq \frac{y^{3}-1}{6} f(y)+\frac{y^{2}}{2} \int_{y}^{\infty} f(u) d u \\
& \geq C y^{3} f(y),
\end{aligned}
$$

where in the fifth line we used the fact that $f(y)$ is decreasing on $(0, \infty)$ and $0<C<$ $\frac{y_{0}^{3}-1}{6 y_{0}^{3}}$ is arbitrary. Now, we have

$$
\begin{aligned}
& \int_{1}^{\infty}\left(\int_{0}^{y} z \nu(\max \{1, z\}, \infty) d z\right)^{-1} d y \\
& =\int_{1}^{y_{0}}\left(\int_{0}^{y} z \nu(\max \{1, z\}, \infty) d z\right)^{-1} d y+\int_{y_{0}}^{\infty}\left(\int_{0}^{y} z \nu(\max \{1, z\}, \infty) d z\right)^{-1} d y \\
& \leq \frac{2\left(y_{0}-1\right)}{y_{0} \int_{y_{0}}^{\infty} f(y) d y}+\frac{1}{C} \int_{y_{0}}^{\infty} \frac{d y}{y^{3} f(y)} \\
& \leq D \int_{1}^{\infty} \frac{d y}{y^{3} f(y)}
\end{aligned}
$$

for some suitably chosen constant $D>0$. Therefore, we have proved the desired result.

Finally, we prove the transience property of a Lévy process with "multiple indices of stability" $\left\{L_{t}\right\}_{t>0}$. Recall that $\left\{L_{t}\right\}_{t>0}$ is a one-dimensional symmetric Lévy process with the Lévy measure $\nu(\{n\})=p_{n}$, where $p_{2 n}=(2 n)^{-\alpha-1}$ and $p_{2 n-1}=(2 n-1)^{-\beta-1}$ for $n \geq 1$ and $\alpha, \beta \in(0, \infty)$. We claim that if $\alpha<1$ and $\beta \geq 1$, then $\left\{L_{t}\right\}_{t \geq 0}$ is transient. Clearly, it suffices to consider the random walk case. Let $\left\{S_{n}\right\}_{n \geq 0}$ be a random walk on $\mathbb{Z}$ with jumps $\mathbb{P}\left(J_{1}=n\right)=c^{-1} p_{n}$, where $c:=\sum_{n \in \mathbb{Z}} p_{n}$ is the norming constant and $p_{n}, n \geq 1$, are as above. First, let us define a sequence of stopping times $\left\{T_{n}\right\}_{n \geq 0}$ inductively by $T_{0}:=0$ and

$$
T_{n}:=\inf \left\{k>T_{n-1}: S_{k} \in 2 \mathbb{Z}\right\},
$$

for $n \geq 1$, and let us prove that $\mathbb{P}\left(T_{n}<\infty\right)=1$ for all $n \geq 1$. We have

$$
\begin{aligned}
\mathbb{P}\left(T_{1}=\infty\right) & =\mathbb{P}\left(S_{k} \in 2 \mathbb{Z}+1 \text { for all } k \geq 1\right) \\
& =\lim _{k \longrightarrow \infty} \mathbb{P}\left(S_{l} \in 2 \mathbb{Z}+1 \text { for all } 1 \leq l \leq k\right) \\
& =\mathbb{P}\left(J_{1} \in 2 \mathbb{Z}+1\right) \lim _{k \longrightarrow \infty}\left(\mathbb{P}\left(J_{1} \in 2 \mathbb{Z}\right)\right)^{k-1}=0 .
\end{aligned}
$$

Now, let us assume that $\mathbb{P}\left(T_{n-1}<\infty\right)=1$ and prove that $\mathbb{P}\left(T_{n}<\infty\right)=1, n \geq 2$. Denote by $N:=T_{n-1}$. Then, by the strong Markov property, we have

$$
\begin{aligned}
\mathbb{P}\left(T_{n}<\infty\right) & =\mathbb{E}\left[1_{\left\{T_{n}<\infty\right\}}\right]=\mathbb{E}\left[1_{\left\{T_{1}<\infty\right\}} \circ \theta_{N}\right]=\mathbb{E}\left[\mathbb{E}\left[1_{\left\{T_{1}<\infty\right\}} \circ \theta_{N} \mid \mathfrak{F}_{N}\right]\right] \\
& =\mathbb{E}\left[\mathbb{E}^{S_{N}}\left[1_{\left\{T_{1}<\infty\right\}}\right]\right]=\sum_{i \in \mathbb{Z}} \mathbb{E}\left[1_{\left\{S_{N}=2 i\right\}}\right]=1,
\end{aligned}
$$


where $\theta_{n}, n \geq 0$, are the shift operators on the canonical state space $\mathbb{Z}^{\{0,1,2, \ldots\}}$ defined by $\left(\theta_{n} \omega\right)(m):=\omega(n+m), m \geq 0$, and $\mathcal{F}_{N}:=\left\{A \in \mathcal{F}: A \cap\{N=n\} \in \sigma\left\{S_{1}, \ldots, S_{n}\right\}\right.$ for all $n \geq 1\}$. Thus, the Markov chain $X_{n}:=S_{T_{n}}$ is well defined. Clearly, $\left\{X_{n}\right\}_{n \geq 0}$ is irreducible on $2 \mathbb{Z}$. Further, note that $\left\{X_{n}\right\}_{n \geq 0}$ and $\left\{S_{n}\right\}_{n \geq 0}$ are transient or recurrent at the same time. Indeed, let us define the following stoping times $\tau:=\inf \left\{n \geq 1: S_{n}=0\right\}$ and $\tilde{\tau}=\inf \left\{n \geq 1: X_{n}=0\right\}$. We have

$$
\mathbb{P}(\tilde{\tau}=\infty)=\mathbb{P}\left(X_{n} \neq 0 \text { for all } n \geq 1\right)=\mathbb{P}\left(S_{n} \neq 0 \text { for all } n \geq 1\right)=\mathbb{P}(\tau=\infty) .
$$

Now, the desired result follows from [6, Propositions 8.1.3 and 8.1.4]. According to this, it suffices to prove the transience property of $\left\{X_{n}\right\}_{n \geq 0}$. Further, note that $\left\{X_{n}\right\}_{n \geq 0}$ is actually a symmetric random walk on $2 \mathbb{Z}$. Indeed, for $n \geq 0$ and $i, j \in \mathbb{Z}$ we have

$$
\begin{aligned}
\mathbb{P} & \left(X_{n+1}=2 j \mid X_{n}=2 i\right) \\
= & \mathbb{P}\left(S_{1}=2 j \mid S_{0}=2 i\right)+\sum_{i_{1} \in \mathbb{Z}} \mathbb{P}\left(S_{1}=2 i_{1}+1 \mid S_{0}=2 i\right) \mathbb{P}\left(S_{2}=2 j \mid S_{1}=2 i_{1}+1\right)+\ldots \\
= & \mathbb{P}\left(S_{1}=2 j-2 i \mid S_{0}=0\right) \\
& +\sum_{i_{1} \in \mathbb{Z}} \mathbb{P}\left(S_{1}=2 i_{1}-2 i+1 \mid S_{0}=0\right) \mathbb{P}\left(S_{2}=2 j-2 i \mid S_{1}=2 i_{1}-2 i+1\right)+\ldots \\
= & \mathbb{P}\left(X_{n+1}=2 j-2 i \mid X_{n}=0\right) \\
= & \mathbb{P}\left(X_{1}=2 j-2 i\right) .
\end{aligned}
$$

Thus, $\left\{X_{n}\right\}_{n \geq 0}$ is spatially homogeneous. Next, for $n, k \geq 0$ and $i, j \in \mathbb{Z}$ we have

$$
\begin{aligned}
\mathbb{P}\left(X_{n+k}-X_{n}=2 i\right) & =\sum_{j \in \mathbb{Z}} \mathbb{P}\left(X_{n+k}=2 i+2 j, X_{n}=2 j\right) \\
& =\sum_{j \in \mathbb{Z}} \mathbb{P}\left(X_{n+k}=2 i+2 j \mid X_{n}=2 j\right) \mathbb{P}\left(X_{n}=2 j\right) \\
& =\mathbb{P}\left(X_{k}=2 i\right),
\end{aligned}
$$

and for $k \geq 1, n_{1}, \ldots, n_{k} \geq 0,0 \leq n_{1} \leq \ldots \leq n_{k}$, and $i_{1}, \ldots, i_{k-1} \in \mathbb{Z}$ we have

$$
\begin{aligned}
& \mathbb{P}\left(X_{n_{k}}-X_{n_{k-1}}=2 i_{k-1}, \ldots, X_{n_{2}}-X_{n_{1}}=2 i_{1}\right) \\
= & \sum_{j \in \mathbb{Z}} \mathbb{P}\left(X_{n_{k}}=2 i_{k-1}+\ldots+2 i_{2}+2 j, \ldots, X_{n_{2}}=2 i_{1}+2 j, X_{n_{1}}=2 j\right) \\
= & \sum_{j \in \mathbb{Z}} \mathbb{P}\left(X_{n_{k}}=2 i_{k-1}+\ldots+2 i_{2}+2 j \mid X_{n_{k-1}}=2 i_{k-2}+\ldots+2 i_{2}+2 j\right) \cdots \\
& \quad \mathbb{P}\left(X_{n_{2}}=2 i_{1}+2 j \mid X_{n_{1}}=2 j\right) \mathbb{P}\left(X_{n_{1}}=2 j\right) \\
= & \mathbb{P}\left(X_{n_{k}-n_{k-1}}=2 i_{k-1}\right) \cdots \mathbb{P}\left(X_{n_{2}-n_{1}}=2 i_{1}\right) \\
= & \mathbb{P}\left(X_{n_{k}}-X_{n_{k-1}}=2 i_{k-1}\right) \cdots \mathbb{P}\left(X_{n_{2}}-X_{n_{1}}=2 i_{1}\right) .
\end{aligned}
$$

Symmetry is trivially satisfied. Thus, the claim follows. Finally, let us show that the random walk $\left\{X_{n}\right\}_{n \geq 0}$ is transient. For $i \in \mathbb{Z} \backslash\{0\}$ we have

$$
\begin{aligned}
\mathbb{P}\left(X_{1}=2 i\right) & =\mathbb{P}\left(S_{1}=2 i\right)+\sum_{j \in \mathbb{Z}} \mathbb{P}\left(S_{1}=2 j+1\right) \mathbb{P}\left(S_{2}=2 i \mid S_{1}=2 j+1\right)+\ldots \\
& \geq \mathbb{P}\left(S_{1}=2 i\right)=c^{-1} p_{2 i}=c^{-1}|2 i|^{-\alpha-1} .
\end{aligned}
$$

Now, since $\alpha<1$, from the condition (1.1) we have

$$
\sum_{n=1}^{\infty} \frac{1}{(2 n)^{3} \mathrm{P}\left(X_{1}=2 n\right)}<\infty
$$

Therefore, we have proved the desired result. 
A transience condition for a class of one-dimensional symmetric Lévy processes

\section{References}

[1] Billingsley, P.: Convergence of probability measures. Second edition. John Wiley \& Sons, New York, 1999. $\mathrm{x}+277$ pp. MR-1700749

[2] Durrett, R.: Probability: theory and examples. Fourth edition. Cambridge University Press, Cambridge, 2010. $\mathrm{x}+428$ pp. MR-2722836

[3] Hobert, J. P. and Schweinsberg, J.: Conditions for recurrence and transience of a Markov chain on $\mathbb{Z}^{+}$and estimation of a geometric success probability. Ann. Statist. 30, (2002), no. 4, 1214-1223. MR-1926175

[4] Jacod, J. and Shiryaev, A. N.: Limit theorems for stochastic processes. Second edition. Springer-Verlag, Berlin, 2003. $\mathrm{xx}+661$ pp. MR-1943877

[5] Lyons, T.: A simple criterion for transience of a reversible Markov chain. Ann. Probab. 11, (1983), no. 2, 393-402. MR-0690136

[6] Meyn, S. P. and Tweedie, R. L.: Markov chains and stochastic stability. Springer-Verlag, London, 1993. xvi+ 548 pp. MR-1287609

[7] Sato, K.: Lévy processes and infinitely divisible distributions. Translated from the 1990 Japanese original. Revised by the author. Cambridge University Press, Cambridge, 1999. xii+486 pp. MR-1739520

[8] Shepp, L. A.: Symmetric random walk. Trans. Amer. Math. Soc. 104, (1962), 144-153. MR0139212

[9] Shepp, L. A.: Recurrent random walks with arbitrarily large steps. Bull. Amer. Math. Soc. 70, (1964), 540-542. MR-0169305

Acknowledgments. The author thanks the anonymous reviewer for careful reading and helpful comments. 


\section{Electronic Journal of Probability Electronic Communications in Probability}

\section{Advantages of publishing in EJP-ECP}

- Very high standards

- Free for authors, free for readers

- Quick publication (no backlog)

\section{Economical model of EJP-ECP}

- Low cost, based on free software (OJS $\left.{ }^{1}\right)$

- Non profit, sponsored by $\mathrm{IMS}^{2}, \mathrm{BS}^{3}, \mathrm{PKP}^{4}$

- Purely electronic and secure (LOCKSS $\left.{ }^{5}\right)$

\section{Help keep the journal free and vigorous}

- Donate to the IMS open access fund ${ }^{6}$ (click here to donate!)

- Submit your best articles to EJP-ECP

- Choose EJP-ECP over for-profit journals

\footnotetext{
${ }^{1}$ OJS: Open Journal Systems http://pkp.sfu.ca/ojs/

2IMS: Institute of Mathematical Statistics http://www.imstat.org/

${ }^{3}$ BS: Bernoulli Society http://www. bernoulli-society.org/

${ }^{4} \mathrm{PK}$ : Public Knowledge Project http://pkp.sfu.ca/

${ }^{5}$ LOCKSS: Lots of Copies Keep Stuff Safe http://www.lockss.org/

${ }^{6}$ IMS Open Access Fund: http://www.imstat.org/publications/open.htm
} 